\title{
Implementation of Analog Modulation Techniques using TMS320C6748 and LabVIEW
}

\author{
${ }^{*}$ Vidyarani K R ${ }^{1}$, Tejaswini $\mathrm{S}^{2}$, Ankitha B $\mathrm{M}^{3}$, Shalini D V ${ }^{4}$ \\ (Dept. of ECE, Channabasaweshwara institute of Technology, Gubbi, Tumakuru, India) \\ Corresponding Author: Vidyarani K R
}

\begin{abstract}
This paper describes Implementation of analog modulation techniques to minimize the error at demodulation using TMS320C6748 kit through Code Composer Studio and LabVIEW. Literature survey clearly shows that few papers described about modulation techniques in LabVIEW only. The main objective of our paper is to perform demodulation using LabVIEW and also realize both modulation and demodulation using C6748 kit. The modulation techniques used for discussion are Amplitude and Frequency Modulation followed by demodulation. The carrier signals are generated and given as an input to modulator for processing. The parameters considered for analysis are modulation index and demodulated signal frequency in both the modulation techniques. The obtained results are compared using both the software's.
\end{abstract}

Keywords: Modulation, TMS320C6748, Code Composer Studio, LabVIEW.

Date of Submission: 01-7-2017

Date of acceptance: 17-07-2017

\section{Introduction}

Communication is the process of conveying message or exchanging information. It means that transmission of the encoded symbols transmitted in the communication channel, at the receiver information is decoded and which recreates the original data [1]. Modulation technique is used in communication process which increases the range of communication, multiplexing and improves quality of reception. It is defined as the process of superimposing the information contents of a base band signal on a carrier signal (which is of high frequency) by varying the characteristic of carrier signal according to the message signal [2]. As we discussed earlier modulation is a very important process in communication systems, because the voice signal is dynamically varying signal hence need a high speed DSP processor to process the signals accurately [3].The modulation schemes are broadly classified into two categories such as analog and digital modulations [4].The topic for implementation is Amplitude \& Frequency modulation followed by demodulation. As literature survey shows that few papers are available to describe about basic voice/signal modulation and demodulation in both LabVIEW and TMS320C6748 DSP kit. TMS320C6748 DSP development kit is a new, robust low-cost development board designed to implement innovative ideas based on the C6748 processor. It is ideal for real time analytic applications, such as finger print recognition, speech processing and face detection [5]. LabVIEW is a development environment for problem solving, accelerated productivity and continual innovation. Hardware integration can be done rapidly which aids to acquire and visualize data sets from virtual I/O devices and real time signals. It is combined with graphical programming syntax that reduces programming time [6]. LabVIEW programs are called Virtual Instruments or VIs, because their appearance and operation imitate physical instruments such as oscilloscopes, multimeters, signal generators and other basic electronic input/output devices [7].

\section{Hardware Description}

The TMS320C6748 fixed and floating point DSP is a low-power application processor based on a C674x DSP core. It includes the C6748 baseboard, SD cards with two demos, BIOS and SDK. The supporting software for this kit is Code Composer Studio (CC Studio) Integrated Development Environment. It as a wide variety of standard interfaces for connectivity and storage, the C6748 kit enables developers to easily bring audio, video and other signals onto the board. Expansion headers allow users to extend the functionality of the kit to include a camera sensor from Leopard Imaging or an LCD screen. Fig.1 shows view of TMS320C6748 development kit. 


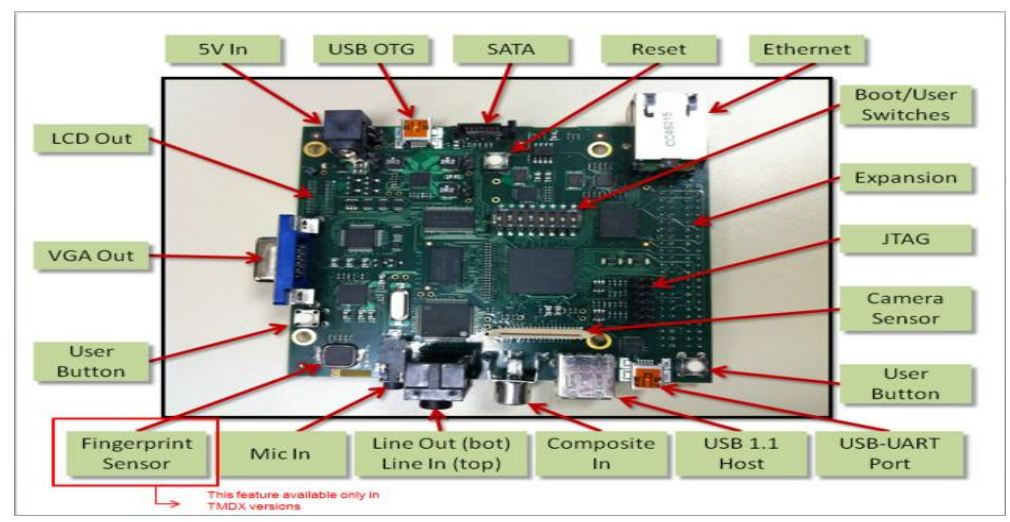

Fig.1: TMS320C6748 development kit

\section{Methodology}

This paper describes the methodology to generate AM and FM signal in CC Studio. The developed algorithm will generate modulation output in TMS320C6748 kit by using baseband signal $\mathrm{m}(\mathrm{t})$ and high frequency carrier signal $\mathrm{c}(\mathrm{t})$. The output of modulations is observed using CRO and the same signals are given as input to another kit (TMS320C6748) for demodulation. The inter connections of the kits and output display equipment connections are as shown in Fig.2. The same can also be implemented in LabVIEW.

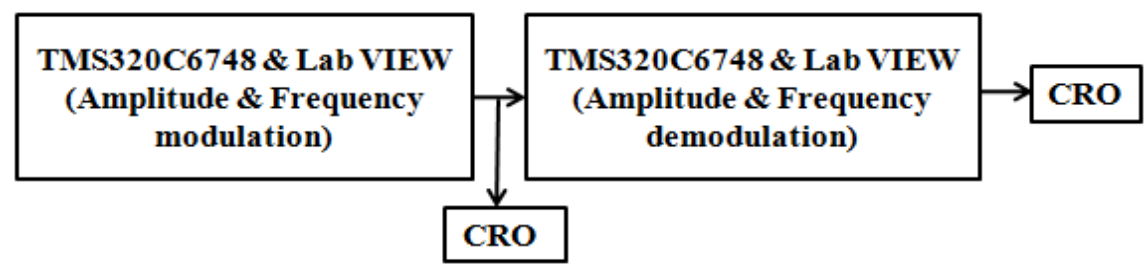

Fig.2: Amplitude / Frequency modulation \& demodulation

\section{Implementation}

The method of implementation is described in this section. Consider the carrier wave of frequency $\left(\mathrm{f}_{\mathrm{c}}=2 \mathrm{kHz}\right)$ and amplitude $\left(\mathrm{A}_{\mathrm{c}}=1 \mathrm{~V}\right)$ given by equation (1):

$$
\begin{aligned}
& c(t)=A_{c} \cos \left(2 \pi f_{c} t\right) \\
& c(t)=\cos (2 * 3.14 * 2 k * t)
\end{aligned}
$$

Let $m(t)$ represents the baseband signal with frequency $\left(f_{m}=500 H z\right)$ and amplitude of $\left(A_{m}=1 V\right)$ shown in equation (2):

$$
\begin{aligned}
& m(t)=A_{m} \cos \left(2 \pi f_{m} t\right) \\
& m(t)=\cos (2 * 3.14 * 500 * t)
\end{aligned}
$$

Amplitude modulation can be achieved when the carrier $c(t)$ is multiplied by the positive quantity $[1+\mathrm{m}(\mathrm{t})]$, which is given by equation (3):

$$
\begin{gathered}
S(t)=[1+m(t)] * c(t) \\
S(t)=A_{c} *\left(1+M * \cos \left(2 \pi f_{m} t\right)\right) * \cos \left(2 \pi f_{c} t\right)
\end{gathered}
$$

Where, $\mathbf{M}=$ modulation index. If $\mathbf{M}<\mathbf{1}$ then $[\mathbf{1}+\mathbf{m}(\mathbf{t})]$ is always positive.

The modulated wave emerges from transmitter [using algorithm as shown in Fig. 3(a) to communication media. At the receiver, the original base band signal is recovered from demodulation procedure. Fig. 3(b) shows the implementation algorithm of amplitude demodulation. It can be realized using envelope detector. Which is achieved using rectifier and filter operation in both the software.

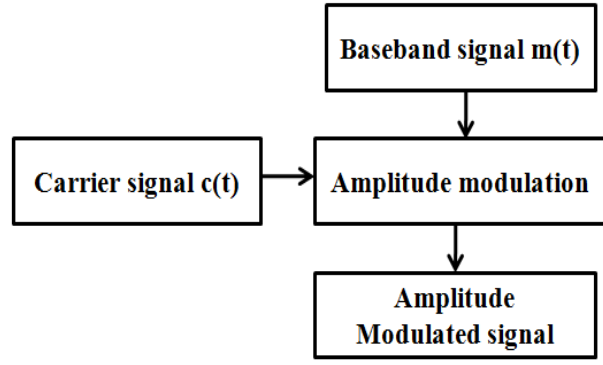

Fig.3 (a): Implementation of Amplitude modulation

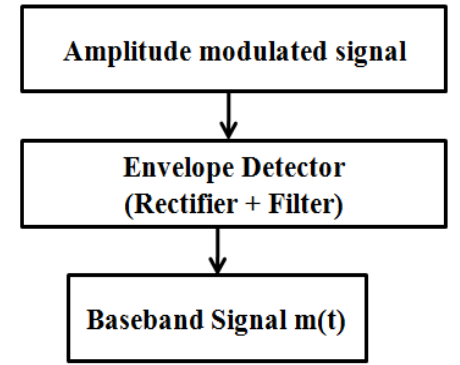

Fig.3 (b): Implementation of Amplitude demodulation 
Frequency modulation can be defined with the help of $\mathrm{c}(\mathrm{t})$ and $\mathrm{m}(\mathrm{t})$ [using (1) and (2)]. We have obtained frequency modulation using equation (4):

$$
S(t)=A_{c} \cos \left(2 \pi f_{c} t+\beta \sin \left(2 \pi f_{m} t\right)\right)
$$

Where, $\boldsymbol{\beta}$ is modulation index. The modulation index relates to variations in the carrier frequency given by equation (5):

$$
\boldsymbol{\beta}=\Delta \mathbf{f} / \mathbf{f}_{\mathbf{m}}
$$

Where $\boldsymbol{\Delta} \mathbf{f}=$ Peak frequency deviation. It is the maximum deviation of the instantaneous frequency from the carrier frequency.

$\mathbf{f}_{\mathbf{m}}=$ The highest frequency component present in the baseband signal $\mathrm{m}(\mathrm{t})$.

If $\boldsymbol{\beta}<<\mathbf{1}$, then the modulation is called narrowband FM, and its bandwidth is approximately $\mathbf{2} \mathbf{f}_{\mathbf{m}}$. Sometimes modulation index $\boldsymbol{\beta}<\mathbf{0 . 3}$ rad is considered as Narrowband FM otherwise Wideband FM.

The frequency demodulation is achieved using differentiator followed by envelope detector. Fig 4(a) \& 4(b) shows the algorithm of implementation for frequency modulation and demodulation.

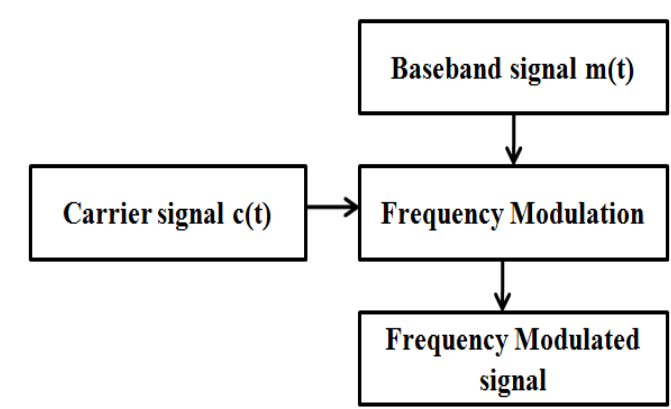

Fig.4 (a): Implementation of Frequency modulation

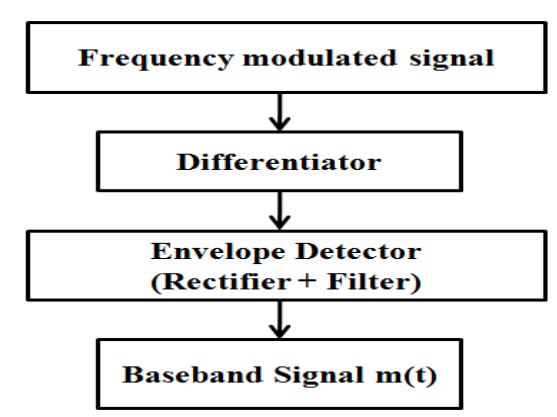

Fig. 4(b): Implementation of Frequency demodulation

\section{Results and discussion}

This section discusses about obtained results. The implementations of AM and FM methods are achieved in both LabVIEW and CC Studio. Fig. 5 (a) and Fig. 6(a) shows the base band signal m(t), amplitude modulated signal and diode output resembles rectification process using $\mathrm{C}$ code. Last stage of demodulation is obtained by filter operation. Realization of filter is achieved by digital IIR filters in C language, since IIR works faster than FIR filters in DSP kits.

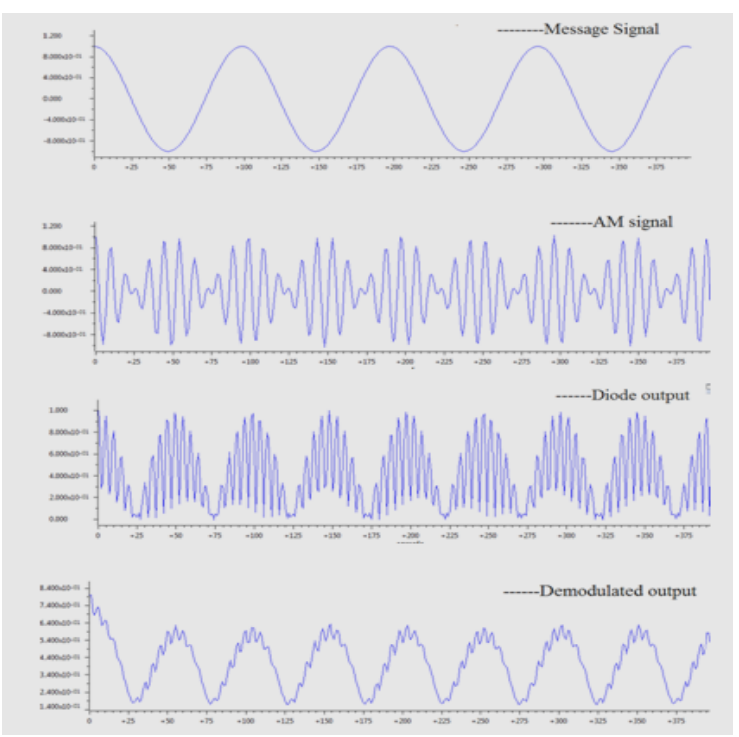

Fig.5 (a): Output waveforms of Amplitude modulation \& demodulation

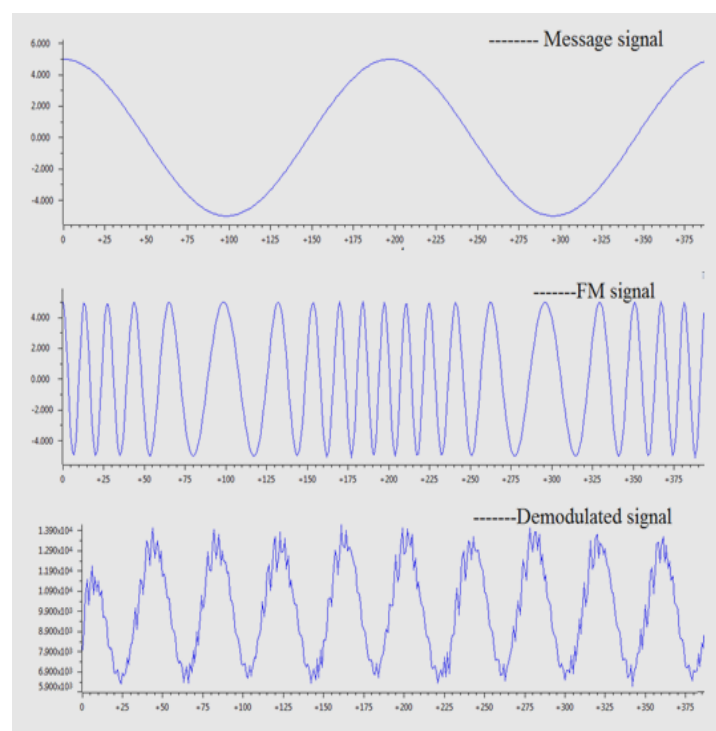

Fig.5 (b): Output waveforms of frequency modulation \& demodulation

Fig. 5(b) and Fig. 6(b) shows output waveforms of frequency modulation and demodulation processes. The baseband signal $\mathrm{m}(\mathrm{t})$ is varied using frequency modulator to obtain FM signal. The demodulation of FM signal is achieved by using many methods; this paper uses differentiator followed by envelope detector. The 
differentiator circuit differentiates FM signal which gives amplitude modulated signal. To do the further demodulation process we followed rectification and filter operation. The reason behind retaining same envelope detector reduces the design complexity.

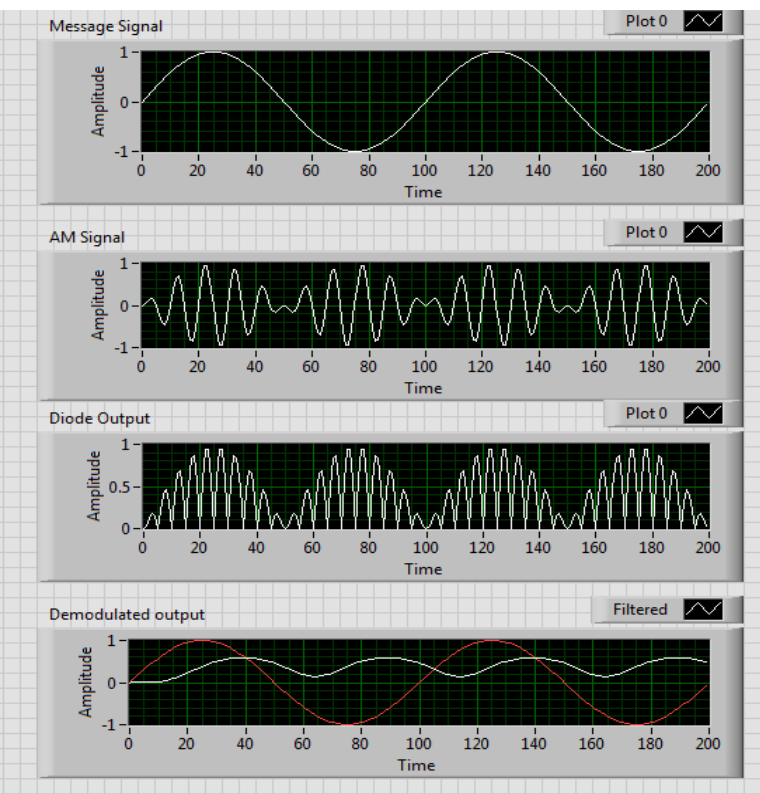

Fig. 6(a): Output waveforms of Amplitude modulation \& demodulation

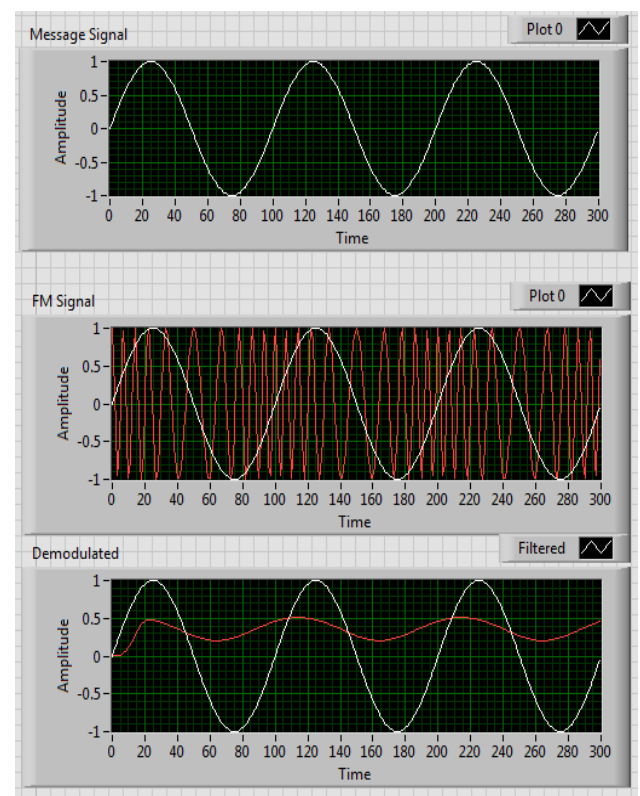

Fig. 6 (b): Output waveforms of frequency modulation \& demodulation

Fig.7 shows the experimental setup of realization of both modulation and demodulation of AM and FM using TMS-C6748 kit. Here one kit exactly resembles transmitter and another kit working as receiver. The communication channel between two systems is double ended stereo cable. Table-I show the performance analysis of CC Studio and LabVIEW based on the few parameters mentioned. Where $\mathrm{M}, \mathrm{f}_{\mathrm{msg}}, \mathrm{f}_{\mathrm{dmod}}$ corresponds to modulation index, message signal frequency and demodulated signal frequency respectively.

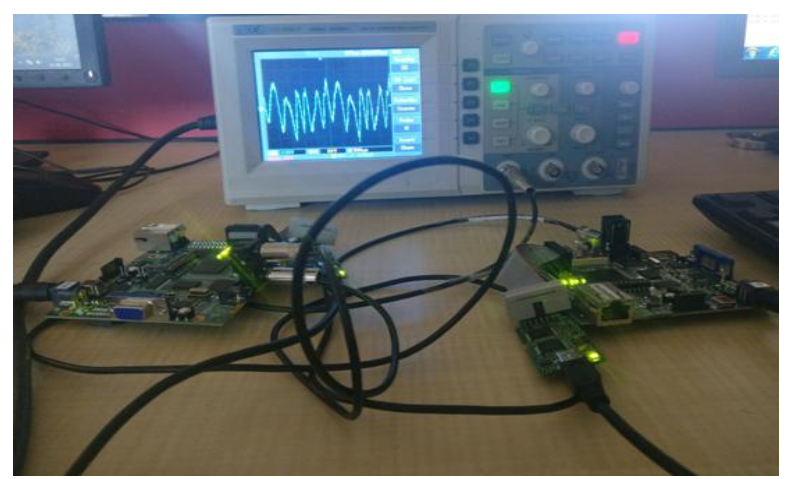

Fig. 7: Experimental setup showing demodulated signal in CRO

Table I: Performance analysis of CC Studio and LabVIEW

\begin{tabular}{|c|c|c|c|c|}
\hline Sl. No. & $\begin{array}{l}\text { Modulation } \\
\text { Techniques }\end{array}$ & Parameters & CC Studio & LabVIEW \\
\hline \multirow{3}{*}{1} & \multirow{2}{*}{$\mathrm{AM}$} & $\mathrm{M}$ & 1 & 1 \\
\cline { 3 - 5 } & & $\mathrm{f}_{\text {msg }}$ & $400 \mathrm{~Hz}$ & $400 \mathrm{~Hz}$ \\
\cline { 3 - 5 } & $\mathrm{f}_{\text {dmod }}$ & $500 \mathrm{~Hz}$ & $400 \mathrm{~Hz}$ \\
\hline \multirow{2}{*}{2} & \multirow{2}{*}{ FM } & $\mathrm{M}$ & 6.6 & 5.1 \\
\cline { 3 - 5 } & & $\mathrm{f}_{\text {msg }}$ & $100 \mathrm{~Hz}$ & $100 \mathrm{~Hz}$ \\
\cline { 3 - 5 } & $\mathrm{f}_{\text {dmod }}$ & $60 \mathrm{~Hz}$ & $100 \mathrm{~Hz}$ \\
\hline
\end{tabular}

\section{Conclusion}

In this paper the implementation of AM and FM modulation techniques are successfully carried out using TMS320C6748 kit through Code Composer Studio and LabVIEW. By observing the results obtained we can conclude that demodulation can be achieved successfully using both the software's. The concept of using both the software's in this paper is that, the person with good programming skill $(\mathrm{C} / \mathrm{C}++)$ can use Code 
Composer Studio and the one who knows system operation well can use LabVIEW, where it provides drag and drop option to the user. The error free output is obtained using LabVIEW and accurate waveforms are obtained using CCS. The future scope includes experiments on digital modulation techniques and real time speech analysis using TMS320C6748 kit.

\section{Acknowledgements}

The authors would like to thank Dr. Suresh D.S, Director, CIT, Gubbi for providing opportunity to work on DSP kits with good infrastructure for financial support under VGST grant. We also express our sign of gratitude to Prof. Sekar R for valuable technical support and suggestions.

\section{References}

[1] Lucky, R.W. Sal, J. Weldon : Principles of Data Communication, McGraw-Hill, New York, 1968.

[2] Schwartz, M.: Information Transmission, Modulation and Noise, McGraw-Hill, New York, 1990.

[3] Chapter 5, Traditional Analog Modulation Techniques, Mikael Olofsson, 2002-2007.

[4] K.Sharma, A.Mishra \& Rajiv Saxena, 'Analog \& Digital Modulation Techniques: An overview', international Journal of Computing Science and Communication Technologies, VOL. 3, NO. 1, July 2010.

[5] TMS320C6748 DSP kit datasheet, "www.ti.com/lit/ds/symlink/tms320c6201.pdf".

[6] C. Uluisik \& L. Sevgi, Electron. \& Communication. Eng. Dept., Dogus Univ., Istanbul, Turkey, 'A LabVIEW-Based Analog Modulation Tool for Virtual and Real Experimentation', IEEE Antenna and Propagation Magazine (Volume : 54, Issue: 6,December 2012).

[7] Korrapati, Raghu B, Swain, Nikunja K, 'Study of Modulation Using Virtual Instruments', Allied Academies International Conference. Academy of Information and Management Sciences.Proceedings;Arden 4.1(2000):78-83.

[8] Ji Shujiao, Zhu Ming, Lei Yanmin, 'The Simulation Design of Communication System Based on Lab VIEW', 2013 2nd International Conference on Measurement, Information and Control.

Vidyarani K R. "Implementation of Analog Modulation Techniques using TMS320C6748 and LabVIEW." IOSR Journal of Research \& Method in Education (IOSR-JRME) 7.4 (2017): 46-50. 